\title{
A Uniformly Accurate Finite-Element Method for a Singularly Perturbed One-Dimensional Reaction-Diffusion Problem
}

\author{
By Eugene O'Riordan and Martin Stynes
}

\begin{abstract}
A finite-element method with exponential basis elements is applied to a selfadjoint, singularly perturbed, two-point boundary value problem. The tridiagonal difference scheme generated is shown to be uniformly second-order accurate for this problem (i.e., the nodal errors are bounded by $C h^{2}$, where $C$ is independent of the mesh size $h$ and the perturbation parameter). With a certain choice of trial functions, uniform first-order accuracy is obtained in $I^{x}[0,1]$.
\end{abstract}

1. Introduction. We consider the following two-point boundary value problem:

$$
\begin{gathered}
L y=\varepsilon^{2}\left(p y^{\prime}\right)^{\prime}-r y=f \quad \text { on }(0,1), \\
y(0)=y_{0}, \quad y(1)=y_{1},
\end{gathered}
$$

where $\varepsilon$ is a parameter in $(0,1], y_{0}$ and $y_{1}$ are given constants, the coefficients $p, r$, and $f$ are in $C^{2}[0,1]$ and satisfy

$$
p(x) \geqslant \xi>0 \text { and } r(x) \geqslant \beta>0 \text { for } x \in[0,1] .
$$

The solution $y(x)$ has, in general, a boundary layer of width $O(\varepsilon)$ at both end points of $[0,1]$.

Letting $y(x)=w(x)(p(x))^{-1 / 2}$, this problem transforms into

$$
\begin{gathered}
\varepsilon^{2} w^{\prime \prime}-d(x) w=g(x), \\
w(0)=y_{0}(p(0))^{1 / 2}, \quad w(1)=y_{1}(p(1))^{1 / 2},
\end{gathered}
$$

where $d(x)$ and $g(x)$ are easily computed.

However, since $d(x)$ involves both first and second derivatives of $p(x)$, a discretization of this transformed problem is not attractive from a numerical point of view. In this paper, we present a numerical method which is directly applicable to problem (1.1).

Finite-difference schemes for singularly perturbed selfadjoint problems of the form (1.2), which are uniformly in $\varepsilon$ accurate, have been examined by various authors. Miller [6] gave sufficient conditions for the uniform first-order convergence of a general three-point difference scheme. Hegarty et al. [4] and Niijima [7] produced uniformly second-order difference schemes. Boglaev [1] examined problem

Received June 20, 1985; revised February 25, 1986.

1980 Mathematics Subject Classification. Primary 65L10. 
(1.2) in a finite-element framework and achieved uniform first-order accuracy at the nodes. Shishkin [11] examined problem (1.1) on a nonuniform mesh, which depends on $\varepsilon$, and obtained convergence results for various difference schemes. A useful discussion of uniform convergence of difference schemes for singularly perturbed two-point boundary value problems is obtained in Doolan, Miller, and Schilders [2]. Schatz and Wahlbin [10] examined problem (1.2) in both one and two dimensions, using a Galerkin finite-element method.

A weak form of problem (1.1) is:

$$
\text { find } y \in H^{1}(0,1) \text { such that }
$$

$$
\begin{aligned}
& B_{\varepsilon}(y, v) \equiv-\varepsilon^{2}\left(p y^{\prime}, v^{\prime}\right)-(r y, v)=(f, v) \quad \text { for all } v \in H_{0}^{1}(0,1), \\
& y(0)=y_{0}, \quad y(1)=y_{1},
\end{aligned}
$$

where (, ) denotes the usual inner product in $L^{2}$.

In this paper, we will discretize (1.3) using a Petrov-Galerkin finite-element method with exponential test functions and a quadrature rule that replaces the functions $p, r$, and $f$ by piecewise-constant approximations. This enables exact evaluation of the modified integrals in (1.3). By introducing a discretized Green's function, the nodal errors are given explicitly in integral form. Our approximation will be shown to be uniformly second-order accurate at the nodes. The crux of the proof lies in suitably bounding the discretized Green's function and its derivative. Using exponential trial functions to interpolate between these nodal values, our approximation is then shown to be uniformly first-order accurate in $L^{\infty}[0,1]$. Thus the second-order nodal accuracy is a superconvergence result.

For a discussion of exponential elements, see Hemker [5], de Groen [3] and the references therein. A discretized Green's function was first used by Stynes and O'Riordan [12] to prove a superconvergence result for a nonselfadjoint singularly perturbed two-point boundary value problem.

Note. Throughout this paper, $C$ will be used to denote a generic constant independent of $x, j, h$, and $\varepsilon$. Also, $g=O\left(h^{p}\right)$ means $|g| \leqslant C h^{p}, p=0,1,2$.

2. The Continuous Problem. The differential operator $L$ in (1.1) satisfies a maximum principle (see Protter and Weinberger [8]). We state this formally as

Lemma 2.1. If $w(0) \leqslant 0, w(1) \leqslant 0, w \in C^{2}[0,1]$ and $L w \geqslant 0$, then $w \leqslant 0$ on $[0,1]$.

In order to obtain the appropriate bounds on the derivatives of the solution of (1.1), we will require some information about the solutions of

$$
L w=g(x, \varepsilon), \quad|w(0)| \leqslant C \text { and }|w(1)| \leqslant C .
$$

We will say that $g$ is of class $(\gamma, j)$ if the derivatives of $g$ with respect to $x$ satisfy

$$
\left|g^{(i)}(x, \varepsilon)\right| \leqslant C\left(1+\varepsilon^{-i} e^{-\gamma x / \varepsilon}+\varepsilon^{-i} e^{-\gamma(1-x) / \varepsilon}\right)
$$

for $0 \leqslant i \leqslant j, x \in[0,1]$, where $\gamma$ is some positive constant independent of $x$ and $\varepsilon$.

LEMMA 2.2. Let $g$ be of class $(\gamma, 0)$. Then the solution $w$ of (2.1) satisfies $|w(x)| \leqslant C$.

Proof. Apply the maximum principle to $-C \pm w(x)$. 
LEMma 2.3. Let $g$ be of class $(\gamma, 0)$. Then the solution $w$ of (2.1) satisfies $\left|w^{\prime}(x)\right| \leqslant C \varepsilon^{-1}$ for $x \in[0,1]$.

Proof. From the differential equation (2.1) we have that

$$
\begin{gathered}
\left(p e^{-D / \varepsilon}\left(w^{\prime}+\varepsilon^{-1}\left((r / p)^{1 / 2}\right) w\right)\right)^{\prime}=\varepsilon^{-2} e^{-D / \varepsilon}\left(g+\left((r p)^{1 / 2}\right)^{\prime} \varepsilon w\right), \\
\left(p e^{D / \varepsilon}\left(w^{\prime}-\varepsilon^{-1}\left((r / p)^{1 / 2}\right) w\right)\right)^{\prime}=\varepsilon^{-2} e^{D / \varepsilon}\left(g-\left((r p)^{1 / 2}\right)^{\prime} \varepsilon w\right),
\end{gathered}
$$

where

$$
D(x)=\int_{t=0}^{x}(r / p)^{1 / 2} d t .
$$

Integrate (2.3) from 0 to $t$, multiply by $p^{-1} e^{2 D / \varepsilon}$ and then integrate again from $t=0$ to $t=1$. Collecting terms, we get $\left|w^{\prime}(0)\right| \leqslant C \varepsilon^{-1}$. By integrating (2.4) from 0 to $x$ and using $\left|w^{\prime}(0)\right| \leqslant C \varepsilon^{-1}$, we get $\left|w^{\prime}(x)\right| \leqslant C \varepsilon^{-1}$.

LEMma 2.4. Let $g$ be of class $(\gamma, 1)$. Then the solution $w$ of (2.1) satisfies

$$
\left|w^{\prime}(x)\right| \leqslant C\left(1+\varepsilon^{-1} \exp (-\delta x / \varepsilon)+\varepsilon^{-1} \exp (-\delta(1-x) / \varepsilon)\right)
$$

for $x \in[0,1]$, where $\delta$ is a positive constant depending only on $p, r$, and $\gamma$.

Proof. Differentiate (2.1) to obtain

$$
\varepsilon^{2} v^{\prime \prime}-(r / p) v=g^{\prime}+r^{\prime} w,
$$

where $v(x)=p w^{\prime}(x)$. Applying the maximum principle to (2.5) with the barrier function

$$
z(x)=C_{1}(\exp (-\delta x / \varepsilon)+\exp (-\delta(1-x) / \varepsilon))+C_{2}
$$

(where $C_{1}, C_{2}$, and $\delta$ are suitably chosen constants) and using Lemma 2.3 to bound $\left|w^{\prime}(0)\right|$ and $\left|w^{\prime}(1)\right|$, we get the desired bound on $\left|w^{\prime}(x)\right|$.

Lemma 2.5. Let $g$ be of class $(\gamma, 2)$. Then the solution $w(x)$ of (2.1) satisfies

$$
\left|w^{\prime \prime}(x)\right| \leqslant C\left(1+\varepsilon^{-2}\{\exp (-\delta x / \varepsilon)+\exp (-\delta(1-x) / \varepsilon)\}\right)
$$

for $x \in[0,1]$, where $\delta$ is a positive constant depending only on $p, r$, and $\gamma$.

Proof. Apply the arguments in Lemmas 2.3 and 2.4 to the differential equation (2.5).

COROLlaRY 2.6. If $y$ is the solution of (1.1), then

$$
\left|y^{(i)}(x)\right| \leqslant C\left(1+\varepsilon^{-i}\left\{\exp \left(-\delta_{0} x / \varepsilon\right)+\exp \left(-\delta_{0}(1-x) / \varepsilon\right)\right\}\right) \quad \text { for } i=0,1,2
$$

and $x \in[0,1]$, where $\delta_{0}$ is some positive constant depending only on $p$ and $r$.

The next lemma gives us an asymptotic expansion for the solution $y$ of (1.1).

LEMMA 2.7. The solution $y(x)$ of (1.1) may be written as

$$
\begin{aligned}
y(x)= & -f(x) / r(x)+K_{1}(\varepsilon) \exp (-D(x) / \varepsilon) \\
& +K_{2}(\varepsilon) \exp (-(D(1)-D(x)) / \varepsilon)+\varepsilon R_{0}(x),
\end{aligned}
$$


where

$$
\begin{aligned}
& \left|K_{1}(\varepsilon)\right| \leqslant C, \quad\left|K_{2}(\varepsilon)\right| \leqslant C, \\
& D(x)=\int_{t=0}^{x}(r(t) / p(t))^{1 / 2} d t, \\
& R_{0}(x) \text { satisfies } L R_{0}(x)=g(x, \varepsilon), \quad R_{0}(0)=R_{0}(1)=0,
\end{aligned}
$$

and $|g(x, \varepsilon)| \leqslant C$ for $x \in[0,1], \varepsilon \in(0,1]$.

Proof. Check by substitution.

The final lemma in this section bounds the derivatives of the remainder term $R_{0}(x)$.

LEMma 2.8. Let $R_{0}(x)$ be defined as in Lemma 2.7; then for $x \in[0,1]$,

$$
\left|R_{0}^{(i)}(x)\right| \leqslant C \varepsilon^{-i}, \quad i=0,1,2 .
$$

Proof. From Lemma 2.2, $\left|R_{0}(x)\right| \leqslant C$. From Lemma 2.3, $\left|R_{0}^{\prime}(x)\right| \leqslant C \varepsilon^{-1}$. Now

$$
\varepsilon^{2}\left(p R_{0}^{\prime}\right)^{\prime}=g(x, \varepsilon)+r R_{0}(x) .
$$

Thus

$$
\left|R_{0}^{\prime \prime}(x)\right| \leqslant C \varepsilon^{-2}, \quad 0 \leqslant x \leqslant 1 .
$$

3. Discretizing the Problem. Divide the interval $[0,1]$ into $N$ subintervals, each of length $h$, and let $x_{j}=j h, 0 \leqslant j \leqslant N$. Define $[0,1]^{*}$ to be $[0,1] \backslash\left\{x_{j}\right\}_{0}^{N}$. As an approximation to the function $r(x)$, define the piecewise-constant function $\bar{r}(x)$ to be

$$
\begin{aligned}
& \bar{r}(x)=\bar{r}_{j}=\left(r\left(x_{j-1}\right)+r\left(x_{j}\right)\right) / 2 \text { for } x \in\left[x_{j-1}, x_{j}\right), j=1, \ldots, N, \\
& \bar{r}\left(x_{N}\right)=\bar{r}_{N} .
\end{aligned}
$$

Define $\bar{p}(x)$ and $\bar{f}(x)$ similarly. Define the operator $\bar{L}$ by

$$
\bar{L} w=\varepsilon^{2}\left(\bar{p} w^{\prime}\right)^{\prime}-\bar{r} w \quad \text { for } w \in C^{2}[0,1],
$$

the derivatives being taken in the sense of distributions.

The next lemma shows that $\bar{L}$ satisfies a maximum principle.

Lemma 3.1. Let $w \in C[0,1] \cap C^{2}\left([0,1]^{*}\right)$ and $\bar{p} w^{\prime} \in C[0,1]$. Suppose $w(0) \leqslant 0$, $w(1) \leqslant 0, \bar{L} w \geqslant 0$ on $[0,1]^{*}$. Then $w \leqslant 0$ on $[0,1]$.

Proof. Suppose the conclusion is false. Then there exists $z \in(0,1)$ such that $\max _{0 \leqslant x \leqslant 1} w(x)=w(z)>0$.

Case (i): $z \in[0,1]^{*}$. Then $w^{\prime \prime}(z)$ exists and $w^{\prime \prime}(z) \leqslant 0$, hence $\bar{L} w(z)=\varepsilon^{2} \bar{p} w^{\prime \prime}(z)$ $-\bar{r} w(z)<0$, a contradiction.

Case (ii): $z=x_{i}$, for some fixed $i$. Since $w \in C[0,1]$, we can choose intervals $\left(c, x_{i}\right)$ and $\left(x_{i}, b\right)$, with $x_{i-1}<c<x_{i}$ and $x_{i}<b<x_{i+1}$, such that $w>0$ on $(c, b)$. Now $\bar{L} w=\varepsilon^{2}\left(\bar{p} w^{\prime}\right)^{\prime}-\bar{r} w \geqslant 0$ on $\left(c, x_{i}\right) \cup\left(x_{i}, b\right)$. Thus $\left(\bar{p} w^{\prime}\right)^{\prime}>0$ on $\left(c, x_{i}\right) \cup$ $\left(x_{i}, b\right)$.

(a) Suppose $\bar{p} w^{\prime}(z) \geqslant 0$. Then, since $\bar{p} w^{\prime} \in C[0,1]$ and $\left(\bar{p} w^{\prime}\right)^{\prime}>0$ on $\left(x_{i}, b\right)$, this implies that $\bar{p} w^{\prime}(x)>0$ on $\left(x_{i}, b\right)$, hence $w^{\prime}(x)>0$ on $\left(x_{i}, b\right)$. Then $w$ cannot have a maximum at $x_{i}$. Contradiction.

(b) Similarly, if $\bar{p} w^{\prime}(z)<0$, it follows that $w^{\prime}<0$ on $\left(c, x_{i}\right)$, hence $w$ cannot have a maximum at $x_{i}$. Contradiction. 
Define each test function $\psi_{k}, k=1, \ldots, N-1$, to be the solution of

$$
\begin{array}{cc}
\bar{L} \psi_{k}=0 & \text { on }[0,1]^{*}, \\
\psi_{k}\left(x_{j}\right)=\delta_{k, j}, & j=0, \ldots, N,
\end{array}
$$

where $\delta_{k, j}$ is the Kronecker delta.

Choose the trial functions $\phi_{i}, i=0, \ldots, N$, to satisfy $\phi_{i}\left(x_{j}\right)=\delta_{i, j}, j=0, \ldots, N$, with the support of each $\phi_{i}(x)$ the one or two subintervals containing $x_{i}$.

The Petrov-Galerkin approximation is

$$
u(x)=\sum_{i=0}^{N} u_{i} \phi_{i}(x),
$$

where the $u_{i}$ are determined from the system of equations

$$
\begin{aligned}
\bar{B}_{\varepsilon}\left(u, \psi_{j}\right)=\left(\bar{f}, \psi_{j}\right), & j=1, \ldots, N-1, \\
u_{0}=y_{0}, & u_{N}=y_{1},
\end{aligned}
$$

where

$$
\bar{B}_{\varepsilon}(v, w) \equiv\left(-\varepsilon^{2} \bar{p} v^{\prime}, w^{\prime}\right)-(\bar{r} v, w), \quad v, w \in H^{1}(0,1)
$$

and

$$
(v, w)=\int_{0}^{1} v(x) w(x) d x, \quad v, w \in L^{2}(0,1) .
$$

LEMMA 3.2. The difference scheme (3.3) has a unique solution.

Proof. The difference scheme (3.3) may be written as

$$
\varepsilon^{2} h^{-2} A u=B f
$$

i.e.,

$$
\varepsilon^{2} h^{-2}\left[a_{j}^{-} u_{j-1}+a_{j}^{0} u_{j}+a_{j}^{+} u_{j+1}\right]=b_{j}^{-} \bar{f}_{j}+b_{j}^{+} \bar{f}_{j+1}, \quad j=1, \ldots, N-1,
$$

where

$$
\begin{aligned}
& a_{j}^{-}=h \bar{p}_{j} \psi_{j}^{\prime}\left(x_{j-1}^{+}\right), \quad a_{j}^{+}=-h \bar{p}_{j+1} \psi_{j}^{\prime}\left(x_{j+1}^{-}\right), \quad a_{j}^{0}=-h \bar{p}_{j} \psi_{j}^{\prime}\left(x_{j}^{-}\right)+h \bar{p}_{j+1} \psi_{j}^{\prime}\left(x_{j}^{+}\right), \\
& b_{j}^{-}=h^{-1}\left(1, \psi_{j}\right)_{j}, \quad b_{j}^{+}=h^{-1}\left(1, \psi_{j}\right)_{j+1} \quad \text { and } \quad(u, v)_{j}=\int_{x_{j-1}}^{x_{j}} u(x) v(x) d x .
\end{aligned}
$$

By explicitly solving for $\psi_{j}(x)$ from (3.1), the reader can easily verify that

$$
a_{j}^{-}>0, \quad a_{j}^{+}>0 \text { and } a_{j}^{0}<-a_{j}^{-}-a_{j}^{+} .
$$

This implies that the matrix $-A$ is an $M$-matrix and hence invertible (see Varga [13]).

REMARK. The difference scheme (3.3) is written out explicitly in Section 7.

4. The Discretized Green's Function. We associate a discretized Green's function $G_{j}(x)$ with each node $x_{j}, j=1, \ldots, N-1$. Define each $G_{j}(x)$ in the sense of distributions by

$$
\begin{gathered}
\bar{L} G_{j}(x)=\varepsilon^{2}\left(\bar{p} G_{j}^{\prime}\right)^{\prime}(x)-\bar{r} G_{j}(x)=\delta\left(x-x_{j}\right), \\
G_{j}(0)=G_{j}(1)=0,
\end{gathered}
$$

where $\delta(\cdot)$ is the Dirac $\delta$-distribution. 
In the classical sense, each $G_{j}(x)$ is defined by

$$
\begin{gathered}
G_{j} \in C[0,1], \\
G_{j}(0)=G_{j}(1)=0, \\
G_{j}^{\prime \prime} \text { exists and is continuous on }[0,1]^{*}, \\
\varepsilon^{2} \bar{p} G_{j}^{\prime \prime}-\bar{r} G_{j}=0 \text { on }[0,1]^{*},
\end{gathered}
$$$$
\lim _{x \rightarrow x_{i}^{+}} \varepsilon^{2} \bar{p} G_{j}^{\prime}(x)-\lim _{x \rightarrow x_{i}^{-}} \varepsilon^{2} \bar{p} G_{j}^{\prime}(x)=\delta_{i, j}, \quad i=1, \ldots, N-1 .
$$

Lemma 4.1. (i) $G_{j}(x)$ is well-defined and lies in the test space spanned by the test functions defined in (3.1).

(ii) $G_{j}(x) \leqslant 0$ on $[0,1]$.

Proof. (i) We must show that there exists a unique choice of $\left\{\lambda_{k}\right\}_{k=1}^{N-1}$ such that

$$
G_{j}(x)=\sum_{k=1}^{N-1} \lambda_{k} \psi_{k}(x)
$$

Now for each $i$ we require

$$
\delta_{i, j}=\left(\phi_{i}, \delta\left(x-x_{j}\right)\right)=\left(\phi_{i}, \bar{L} G_{j}\right)=\bar{B}_{\varepsilon}\left(\phi_{i}, G_{j}\right)=\sum_{k=1}^{N-1} \lambda_{k} \bar{B}_{\varepsilon}\left(\phi_{i}, \psi_{k}\right) .
$$

Thus, we have the system of equations

$$
\sum_{k=1}^{N-1} \lambda_{k} \bar{B}_{\varepsilon}\left(\phi_{i}, \psi_{k}\right)=\delta_{i, j}, \quad i=1, \ldots, N-1,
$$

for the unknowns $\lambda_{1}, \ldots, \lambda_{N-1}$. The matrix of this system is the transpose of the matrix associated with the difference scheme (3.3), which from Lemma 3.2 is known to be invertible.

(ii) The matrix in (3.3) is the negative of an $M$-matrix, so its inverse has all nonpositive entries (see Varga [13]). Thus $\lambda_{k} \leqslant 0, k=1, \ldots, N-1$. Now $\psi_{k}>0$ on $\left(x_{k-1}, x_{k+1}\right)$. Since $G_{j}(x)=\sum_{k=1}^{N-1} \lambda_{k} \psi_{k}(x)$, we have $G_{j} \leqslant 0$ on $[0,1]$.

THEOREM 4.2. For $x \in[0,1], \quad\left|G_{j}(x)\right| \leqslant C \varepsilon^{-1} \exp \left(-\gamma\left|x-x_{j}\right| / \varepsilon\right)$, where $\gamma=$ $\min \left((r(x) p(x))^{1 / 2}\right) / \max (p(x))$.

Proof. Set $\omega(x)=-\left\{\exp \left(-(\beta \xi)^{1 / 2}\left|x-x_{j}\right| /(\bar{p}(x) \varepsilon)\right)\right\} /\left(2(\beta \xi)^{1 / 2} \varepsilon\right)$. Note that $\bar{p} \omega^{\prime}\left(x_{j}^{-}\right)=-1 /\left(2 \varepsilon^{2}\right), \bar{p} \omega^{\prime}\left(x_{j}^{+}\right)=1 /\left(2 \varepsilon^{2}\right)$. So $\bar{p} \omega^{\prime}(x)$ has a jump of $\varepsilon^{-2}$ at $x_{j}$. Let $z=\omega-G_{j}$ on $[0,1]$. Then $z \in C[0,1], z \in C^{2}\left([0,1]^{*}\right)$ and $\bar{p} z^{\prime} \in C[0,1]$ (jumps cancel at $x=x_{j}$ ). Thus we are able to apply the maximum principle for $\bar{L}$, given in Lemma 3.1. Now $z(0)<0$ and $z(1)<0$. On $[0,1]^{*}$,

$$
\bar{L} z=\varepsilon^{2}\left(\bar{p} \omega^{\prime}\right)^{\prime}-(\beta \xi \omega) / \bar{p}+(\beta \xi / \bar{p}-\bar{r}) \omega-\bar{L} G_{j}=(\beta \xi-\bar{r} \bar{p}) \omega / \bar{p} \geqslant 0 .
$$

By Lemma $3.1, z \leqslant 0$ on $[0,1]$. Thus $G_{j}(x) \geqslant \omega(x)$, but from Lemma $4.1 G_{j}(x) \leqslant 0$. Thus $\left|G_{j}(x)\right| \leqslant|\omega(x)|$. The result follows.

The next result bounds the derivative of the Green's function.

THEOREM 4.3. $\left|G_{j}^{\prime}(t)\right| \leqslant C \varepsilon^{-2} \exp \left(-\gamma\left|t-x_{j}\right| / \varepsilon\right)$ for $t \in[0,1] \backslash\left\{x_{j}\right\}$, where $\gamma$ is as in Theorem 4.2 . 
Proof. From the definition of $G_{j}(t)$ in (4.1), we have

$$
\begin{aligned}
& \left(\bar{p} \exp (-\bar{D} / \varepsilon)\left[G_{j}^{\prime}+(\bar{r} / \bar{p})^{1 / 2} \varepsilon^{-1} G_{j}^{\prime}\right]\right)^{\prime} \\
& \quad=\varepsilon^{-2} \exp (-\bar{D} / \varepsilon)\left[\delta\left(x-x_{j}\right)+(\sqrt{\overline{r p}})^{\prime} \varepsilon G_{j}\right], \\
& \left(\bar{p} \exp (\bar{D} / \varepsilon)\left[G_{j}^{\prime}-(\bar{r} / \bar{p})^{1 / 2} \varepsilon^{-1} G_{j}^{\prime}\right]\right)^{\prime} \\
& =\varepsilon^{-2} \exp (\bar{D} / \varepsilon)\left[\delta\left(x-x_{j}\right)-(\sqrt{\overline{r p}})^{\prime} \varepsilon G_{j}\right],
\end{aligned}
$$

where $\bar{D}(x)=\int_{0}^{x}(\bar{r}(t) / \bar{p}(t))^{1 / 2} d t$.

Integrate (4.3) from 0 to $t$, multiply by $(\bar{p})^{-1} \exp (2 \bar{D} / \varepsilon)$ and then integrate again from $t=0$ to $t=1$. Collecting terms, we get

$$
\left|G_{j}^{\prime}(0)\right| \leqslant C \varepsilon^{-2} \exp \left(-\bar{D}\left(x_{j}\right) / \varepsilon\right)+C \varepsilon^{-2}|I|,
$$

where

$$
I=\int_{0}^{1}(\varepsilon \bar{p})^{-1} \exp (-2(\bar{D}(1)-\bar{D}(t)) / \varepsilon) \int_{0}^{t} \exp (-\bar{D}(s) / \varepsilon) \varepsilon G_{j}(\sqrt{\overline{r p}})^{\prime} d s d t .
$$

The integral $I$ equals

$$
\begin{aligned}
& \sum_{i=1}^{N} \sum_{k=1}^{i-1} \int_{x_{i-1}}^{x_{i}}(\bar{p} \varepsilon)^{-1} \exp (-2(\bar{D}(1)-\bar{D}(t)) / \varepsilon) \exp \left(-\bar{D}\left(x_{k}\right) / \varepsilon\right) \\
& \times \varepsilon G_{j}\left(x_{k}\right)\left\{\sqrt{\overline{r p}}\left(x_{k}^{+}\right)-\sqrt{\overline{r p}}\left(x_{k}^{-}\right)\right\} d t .
\end{aligned}
$$

Now for any set of numbers $\left\{b_{i k}\right\}$, it is easy to show by induction on $N$ that

$$
\sum_{i=1}^{N} \sum_{k=1}^{i-1} b_{i k}=\sum_{k=1}^{N-1} \sum_{i=k+1}^{N} b_{i k}
$$

Applying this above yields

$$
\begin{aligned}
I= & \sum_{k=1}^{N-1} \exp \left(-\bar{D}\left(x_{k}\right) / \varepsilon\right) \varepsilon G_{j}\left(x_{k}\right)\left\{\sqrt{\overline{r p}}\left(x_{k}^{+}\right)-\sqrt{\overline{r p}}\left(x_{k}^{-}\right)\right\} \\
& \times \sum_{i=k+1}^{N} \int_{x_{i-1}}^{x_{i}}(\bar{p} \varepsilon)^{-1} \exp (-2(\bar{D}(1)-\bar{D}(t)) / \varepsilon) d t .
\end{aligned}
$$

Thus,

$$
|I| \leqslant \sum_{k=1}^{N-1} C h \exp \left(-\gamma x_{k} / \varepsilon\right) \varepsilon\left|G_{j}\left(x_{k}\right)\right| \int_{x_{k}}^{1} \varepsilon^{-1} \exp (-2(\bar{D}(1)-\bar{D}(t)) / \varepsilon) d t .
$$

Bounding each integral in the sum by $C$ and using the bound on $\left|G_{j}\left(x_{k}\right)\right|$ from Theorem 4.2, we get

$$
\begin{aligned}
|I| & \leqslant C h\left[\sum_{k=1}^{j} \exp \left(-\gamma x_{j} / \varepsilon\right)+\sum_{k=j+1}^{N-1} \exp \left(-\gamma\left(2 x_{k}-x_{j}\right) / \varepsilon\right)\right] \\
& \leqslant C h \sum_{k=1}^{N-1} \exp \left(-\gamma x_{j} / \varepsilon\right) \leqslant C \exp \left(-\gamma x_{j} / \varepsilon\right), \quad \text { since } h N=1 .
\end{aligned}
$$

We have thus shown that

$$
\left|G_{j}^{\prime}(0)\right| \leqslant C \varepsilon^{-2} \exp \left(-\gamma x_{j} / \varepsilon\right) .
$$


We will use this to bound $G_{j}^{\prime}(t)$ on $\left[0, x_{j}\right)$. Let $t \in\left[x_{i-1}, x_{i}\right)$, where $i \leqslant j$. Integrate (4.4) from 0 to $t$ and multiply by $(\bar{p}(t))^{-1} \exp (-\bar{D}(t) / \varepsilon)$. Thus, collecting terms, we get

$$
\begin{aligned}
\left|G_{j}^{\prime}(t)\right| \leqslant & C \varepsilon^{-2} \exp \left(-\gamma\left(x_{j}-t\right) / \varepsilon\right) \\
& +C \varepsilon^{-2}\left|\int_{0}^{t} \exp (-(\bar{D}(t)-\bar{D}(s)) / \varepsilon) \varepsilon G_{j}(s)(\sqrt{\overline{r p}})^{\prime}(s) d s\right| \\
\leqslant & C \varepsilon^{-2} \exp \left(-\gamma\left(x_{j}-t\right) / \varepsilon\right) \\
& +C h \varepsilon^{-2} \sum_{k=1}^{i-1} \exp \left(-\gamma\left(t-x_{j}\right) / \varepsilon\right) \exp \left(-\gamma\left(x_{j}-x_{k}\right) / \varepsilon\right) \\
= & C \varepsilon^{-2} \exp \left(-\gamma\left(x_{j}-t\right) / \varepsilon\right) \\
& +C h \varepsilon^{-2} \exp \left(-\gamma\left(x_{j}-t\right) / \varepsilon\right) \sum_{k=1}^{i-1} \exp \left(-2 \gamma\left(t-x_{k}\right) / \varepsilon\right) \\
\leqslant & C \varepsilon^{-2} \exp \left(-\gamma\left(x_{j}-t\right) / \varepsilon\right) .
\end{aligned}
$$

By symmetry arguments, we now extend this bound to $\left(x_{j}, 1\right]$. For $0 \leqslant x \leqslant 1$, set

$$
F_{j}(x)=G_{j}(1-x), \quad \bar{r}_{1}(x)=\bar{r}(1-x) \quad \text { and } \quad \bar{p}_{1}(x)=\bar{p}(1-x) .
$$

Then

$$
\begin{aligned}
\varepsilon^{2}\left(\bar{p}_{1} F_{j}^{\prime}\right)^{\prime}(x)-\bar{r}_{1}(x) F_{j}(x) & =\varepsilon^{2}\left(\bar{p} G_{j}^{\prime}\right)^{\prime}(1-x)-\bar{r}(1-x) G_{j}(1-x) \\
& =\delta\left(1-x-x_{j}\right)=\delta\left(x-\left(1-x_{j}\right)\right) .
\end{aligned}
$$

That is, $F_{j}$ satisfies the same sort of equation as (4.1) (of course $F_{j}(0)=F_{j}(1)=0$ ). Note, however, that we have $\delta\left(x-\left(1-x_{j}\right)\right)$ instead of $\delta\left(x-x_{j}\right)$, as we had for $G_{j}(x)$. From (4.6), we therefore have

$$
\left|G_{j}^{\prime}(1-y)\right|=\left|F_{j}^{\prime}(y)\right| \leqslant C \varepsilon^{-2} \exp \left(-\gamma\left(1-x_{j}-y\right) / \varepsilon\right), \quad 0 \leqslant y<1-x_{j} .
$$

Let $x=1-y$. Then

$$
\left|G_{j}^{\prime}(x)\right| \leqslant C \varepsilon^{-2} \exp \left(-\gamma\left(x-x_{j}\right) / \varepsilon\right) \text { for } x_{j}<x \leqslant 1,
$$

which together with (4.6) completes the proof.

Corollary 4.4. (a) $\left|G_{j}\left(x_{i}\right)-G_{j}\left(x_{i-1}\right)\right| \leqslant C h \varepsilon^{-2} \exp \left(-\gamma\left(x_{j}-x_{i}\right) / \varepsilon\right)$ for $1 \leqslant i \leqslant j$.

(b) $\left|G_{j}\left(x_{i}\right)-G_{j}\left(x_{i-1}\right)\right| \leqslant C h \varepsilon^{-2} \exp \left(-\gamma\left(x_{i-1}-x_{j}\right) / \varepsilon\right)$ for $j<i \leqslant N$.

Proof. (a)

$$
\begin{aligned}
\left|G_{j}\left(x_{i}\right)-G_{j}\left(x_{i-1}\right)\right| & =h\left|G_{j}^{\prime}\left(\eta_{i}\right)\right|, \quad x_{i-1}<\eta_{i}<x_{i}, \\
& \leqslant C h \varepsilon^{-2} \exp \left(-\gamma\left(x_{j}-\eta_{i}\right) / \varepsilon\right) \quad \text { for } i \leqslant j, \\
& \leqslant C h \varepsilon^{-2} \exp \left(-\gamma\left(x_{j}-x_{i}\right) / \varepsilon\right), \quad \text { since } \eta_{i}<x_{i} .
\end{aligned}
$$

(b) Similarly.

5. Estimating the Nodal Error. The error at each node $x_{j}$ can be given explicitly in terms of the discretized Green's function. 
THEOREM 5.1. Let $y(x)$ be the solution of problem (1.1). Let $u(x)$ be our approximation defined in (3.2) and (3.3). Then

$$
\begin{aligned}
y\left(x_{j}\right)-u\left(x_{j}\right)=\left(f-\bar{f}, G_{j}\right)+\left(\varepsilon^{2}(p-\bar{p}) y^{\prime}, G_{j}^{\prime}\right)+\left(r-\bar{r}, y G_{j}\right) & \\
& \quad \text { for } j=1, \ldots, N-1 .
\end{aligned}
$$

Proof.

$$
\begin{aligned}
y\left(x_{j}\right)-u\left(x_{j}\right) & =\left(y-u, \delta\left(x-x_{j}\right)\right) \\
& =\left(y-u, \bar{L} G_{j}\right)=\bar{B}_{\varepsilon}\left(y-u, G_{j}\right)=\bar{B}_{\varepsilon}\left(y, G_{j}\right)-\bar{B}_{\varepsilon}\left(u, G_{j}\right) \\
& =\bar{B}_{\varepsilon}\left(y, G_{j}\right)-\left(\bar{f}, G_{j}\right), \quad \text { by }(3.3) \text { and Lemma 4.1(i) } \\
& =B_{\varepsilon}\left(y, G_{j}\right)+\left(\varepsilon^{2}(p-\bar{p}) y^{\prime}, G_{j}^{\prime}\right)+\left(r-\bar{r}, y G_{j}\right)-\left(\bar{f}, G_{j}\right) \\
& =\left(f, G_{j}\right)-\left(\bar{f}, G_{j}\right)+\left(\varepsilon^{2}(p-\bar{p}) y^{\prime}, G_{j}^{\prime}\right)+\left(r-\bar{r}, y G_{j}\right) .
\end{aligned}
$$

On the interval $\left[x_{i-1}, x_{i}\right]$ we may write the Green's function in the form

$$
G_{j}(x)=a_{i} \sinh \left(\alpha_{i}\left(x-x_{i-0.5}\right) / h\right)+b_{i} \cosh \left(\alpha_{i}\left(x-x_{i-0.5}\right) / h\right),
$$

where

$$
\begin{aligned}
a_{i} & =\left(G_{j}\left(x_{i}\right)-G_{j}\left(x_{i-1}\right)\right) /\left(2 \sinh \left(\alpha_{i} / 2\right)\right), \\
b_{i} & =\left(G_{j}\left(x_{i}\right)+G_{j}\left(x_{i-1}\right)\right) /\left(2 \cosh \left(\alpha_{i} / 2\right)\right), \\
x_{i-0.5} & =\left(x_{i-1}+x_{i}\right) / 2 \text { and } \alpha_{i}=\left(\bar{r}_{i} / \bar{p}_{i}\right)^{1 / 2} h \varepsilon^{-1} .
\end{aligned}
$$

The following term will arise repeatedly throughout the error analysis:

$$
\tau_{i} \equiv \int_{x_{i-1}}^{x_{i}}\left[\left(x-x_{i-0.5}\right) \sinh \left(\alpha_{i}\left(x-x_{i-0.5}\right) / h\right)\right] /\left[2 \sinh \left(\alpha_{i} / 2\right)\right] d x .
$$

The next lemma is a technical lemma needed in the analysis of Lemma 5.3.

LEMMA 5.2 (i) $\left|\tau_{i}\right| \leqslant C h^{2}$,

(ii) $\left|\tau_{i}\right| \leqslant C h \varepsilon$,

(iii) $\left|\tau_{i+1}-\tau_{i}\right| \leqslant C h^{2} \varepsilon$,

(iv) $\left|\tau_{i}-\tau_{j}\right| \leqslant C h^{2}\left|x_{i}-x_{j}\right|$

where $\tau_{i}$ is defined in (5.3) and $1 \leqslant i, j \leqslant N$.

Proof. (i) Setting $y=2\left(x-x_{i-0.5}\right) / h$,

$$
\tau_{i}=\left(h^{2} / 8\right) \int_{-1}^{1}\left[y \sinh \left(\alpha_{i} y / 2\right)\right] /\left[\sinh \left(\alpha_{i} / 2\right)\right] d y .
$$

Thus $\left|\tau_{i}\right| \leqslant C h^{2}$.

(ii) Evaluating the integral in (i), we get

$$
\left|\tau_{i}\right|=\left|\left(h / \alpha_{i}\right)^{2}\left\{\left(\alpha_{i} / 2\right) \operatorname{coth}\left(\alpha_{i} / 2\right)-1\right\}\right| \leqslant C h \varepsilon,
$$

since $|x \operatorname{coth}(x)-1| \leqslant C x$ for all $x$.

(iii) Using (ii), we get

$$
\begin{aligned}
\tau_{i+1}-\tau_{i}= & h^{2}\left(\alpha_{i+1}^{-2}-\alpha_{i}^{-2}\right)\left\{\left(\alpha_{i+1} / 2\right) \operatorname{coth}\left(\alpha_{i+1} / 2\right)-1\right\} \\
& +h^{2} \alpha_{i}^{-2}\left\{\left(\alpha_{i+1} / 2\right) \operatorname{coth}\left(\alpha_{i+1} / 2\right)-\left(\alpha_{i} / 2\right) \operatorname{coth}\left(\alpha_{i} / 2\right)\right\} .
\end{aligned}
$$


Now $|x \operatorname{coth}(x)-1| \leqslant C x$ and $|x \operatorname{coth}(x)-y \operatorname{coth}(y)| \leqslant|x-y|$, since $\left|(x \operatorname{coth}(x))^{\prime}\right|$ $\leqslant 1$ for all $x$. Thus $\left|\tau_{i+1}-\tau_{i}\right| \leqslant C h^{2} \varepsilon$.

(iv) Fix $i, j \in\{1, \ldots, N\}$. Let

$$
u(y)=\sinh \left(\alpha_{i} y / 2\right) / \sinh \left(\alpha_{i} / 2\right) \text { and } v(y)=\sinh \left(\alpha_{j} y / 2\right) / \sinh \left(\alpha_{j} / 2\right),
$$

$0 \leqslant y \leqslant 1$. Let $M_{i} w=w^{\prime \prime}-\left(\alpha_{i}^{2} / 4\right) w$, for $w \in C^{2}[0,1]$. Note that $M_{i}$ satisfies a maximum principle and $M_{i} u=0$. Apply the maximum principle for $M_{i}$ to

$$
z(y)=-C\left|x_{i}-x_{j}\right| \pm(u-v)(y), \quad 0 \leqslant y \leqslant 1 .
$$

Now $z(0)=z(1)=-C\left|x_{i}-x_{j}\right| \leqslant 0$ and

$$
M_{i} z=C\left(\alpha_{i}^{2} / 4\right)\left|x_{i}-x_{j}\right| \pm\left(\alpha_{i}^{2}-\alpha_{j}^{2}\right) v / 4 \geqslant 0
$$

for suitably large $C$, since $|v| \leqslant 1$. Thus $z \leqslant 0$. That is, $|u-v| \leqslant C\left|x_{i}-x_{j}\right|$. Thus

$$
\left|\tau_{i}-\tau_{j}\right|=\left(h^{2} / 4\right)\left|\int_{0}^{1} y(u(y)-v(y)) d y\right| \leqslant C h^{2}\left|x_{i}-x_{j}\right| .
$$

Note that

$$
\int_{x_{i-1}}^{x_{i}}\left(x-x_{i-0.5}\right) \cosh \left(\alpha_{i}\left(x-x_{i-0.5}\right) / h\right) d x=0,
$$

since the integrand is an odd function about $x=x_{i-0.5}$.

LEMMA 5.3 Let $G_{j}(x)$ be the solution of (4.1). If $k, q$, and $w$ are functions defined on $[0,1]$ such that $\left|k^{(i)}\right| \leqslant C$ for $i=0,1,2,\left|q^{(i)}\right| \leqslant C$ for $i=0,1,\left|w^{(i)}\right| \leqslant C \varepsilon^{-i}$ for $i=0,1, \bar{k}(x)=\left(k\left(x_{i}\right)+k\left(x_{i-1}\right)\right) / 2$ for $x \in\left[x_{i-1}, x_{i}\right)$ and $D(x)=$ $\int_{t=0}^{x}(r(t) / p(t))^{1 / 2} d t$, then

(i) $\left|\left(k-\bar{k}, q G_{j}\right)\right| \leqslant C h^{2}$,

(ii) $\left|\left(k-\bar{k}, q \exp (-D / \varepsilon) G_{j}\right)\right| \leqslant C h^{2}$,

(iii) $\left|\left(k-\bar{k}, q \exp (-(D(1)-D(x)) / \varepsilon) G_{j}\right)\right| \leqslant C h^{2}$,

(iv) $\left|\left(k-\bar{k}, \varepsilon^{2} q G_{j}^{\prime}\right)\right| \leqslant C h^{2}$,

(v) $\left|\left(k-\bar{k}, \varepsilon q \exp (-D / \varepsilon) G_{j}^{\prime}\right)\right| \leqslant C h^{2}$,

(vi) $\left|\left(k-\bar{k}, \varepsilon q \exp (-(D(1)-D(x)) / \varepsilon) G_{j}^{\prime}\right)\right| \leqslant C h^{2}$,

(vii) $\left|\left(k-\bar{k}, \varepsilon^{2} w G_{j}^{\prime}\right)\right| \leqslant C h^{2}$.

Proof. For $x \in\left[x_{i-1}, x_{i}\right)$,

$k(x)-\bar{k}(x)=k^{\prime}\left(x_{i-0.5}\right)\left(x-x_{i-0.5}\right)+O\left(h^{2}\right), \quad q(x)=q\left(x_{i-0.5}\right)+O(h)$.

Let $s\left(x_{i}\right)=k^{\prime}\left(x_{i-0.5}\right) q\left(x_{i-0.5}\right)$ and set

$$
(u, v)_{i}=\int_{x_{i-1}}^{x_{i}} u(x) v(x) d x \quad \text { for } u, v \in L^{2}[0,1] .
$$

(i) Theorem 4.2 implies that $\left(1,\left|G_{j}\right|\right) \leqslant C$. Using this and (5.2), (5.3), and (5.4), we have

$$
\begin{aligned}
\left(k-\bar{k}, q G_{j}\right) & =\sum_{i=1}^{N} s\left(x_{i}\right) \tau_{i}\left(G_{j}\left(x_{i}\right)-G_{j}\left(x_{i-1}\right)\right)+O\left(h^{2}\right) \\
& =\sum_{i=1}^{N-1}\left(\xi_{i}-\xi_{i-1}\right) G_{j}\left(x_{i}\right)+O\left(h^{2}\right),
\end{aligned}
$$

where we put $\xi_{i}=s\left(x_{i}\right) \tau_{i}$ and used $G_{j}\left(x_{0}\right)=G_{j}\left(x_{N}\right)=0$. By Lemma 5.2, we have

$$
\begin{aligned}
\left|\xi_{i}-\xi_{i+1}\right| & \leqslant\left|s\left(x_{i}\right)-s\left(x_{i+1}\right)\right|\left|\tau_{i}\right|+\left|s\left(x_{i+1}\right)\right|\left|\tau_{i}-\tau_{i+1}\right| \\
& \leqslant C h \min \left(h^{2}, h \varepsilon\right)+C \min \left(h^{3}, h^{2} \varepsilon\right) \leqslant C h^{2} \min (h, \varepsilon) .
\end{aligned}
$$


Combining this with the estimate $\left|G_{j}\left(x_{i}\right)\right| \leqslant C \varepsilon^{-1} \exp (-\gamma h|j-i| / \varepsilon)$, which follows from Theorem 4.2, we get

$$
\begin{aligned}
\left|\sum_{i=1}^{N}\left(\xi_{i}-\xi_{i+1}\right) G_{j}\left(x_{i}\right)\right| & \leqslant C h^{2} \min (h / \varepsilon, 1) \sum_{i=1}^{N-1} \exp (-\gamma h|j-i| / \varepsilon) \\
& \leqslant C h^{2} \min (\lambda, 1) /\left(1-e^{-\gamma \lambda}\right)
\end{aligned}
$$

with $\lambda=h / \varepsilon$. Since $\lambda /\left(1-e^{-\gamma \lambda}\right) \leqslant C$ for $0<\lambda \leqslant 1$ and $1 /\left(1-e^{-\gamma \lambda}\right) \leqslant C$ for $\lambda>1$, we have

$$
\left|\sum_{i=1}^{N}\left(\xi_{i}-\xi_{i+1}\right) G_{j}\left(x_{i}\right)\right| \leqslant C h^{2}
$$

which completes the proof of (i).

(ii) Let $\bar{D}(x)=\int_{t=0}^{x}(\bar{r}(t) / \bar{p}(t))^{1 / 2} d t$. We have

$$
\left(k-\bar{k}, q e^{-D / \varepsilon} G_{j}\right)=\left(k-\bar{k}, q \exp (-\bar{D} / \varepsilon) G_{j}\right)+O\left(h^{2}\right),
$$

since $|k-\bar{k}| \leqslant C h,\left(1,\left|G_{j}\right|\right) \leqslant C,|q| \leqslant C$ and

$$
|\exp (-D(x) / \varepsilon)-\exp (-\bar{D}(x) / \varepsilon)| \leqslant C h x \varepsilon^{-1} \exp (-\gamma x / \varepsilon) \leqslant C h .
$$

Hence,

$$
\begin{aligned}
(k-\bar{k} & \left., q \exp (-\bar{D} / \varepsilon) G_{j}\right) \\
& =\sum_{i=1}^{N} s\left(x_{i}\right) \exp \left(-\bar{D}\left(x_{i-0.5}\right) / \varepsilon\right)\left(x-x_{i-0.5}, e^{-\alpha_{i}\left(x-x_{i-0.5}\right) / h} G_{j}\right)_{i}+O\left(h^{2}\right) .
\end{aligned}
$$

On the interval $\left[x_{i-1}, x_{i}\right)$,

$$
\begin{aligned}
G_{j}(x)= & \left\{\left(a_{i}+b_{i}\right) / 2\right\} \exp \left(\alpha_{i}\left(x-x_{i-0.5}\right) / h\right) \\
& +\left\{\left(b_{i}-a_{i}\right) / 2\right\} \exp \left(-\alpha_{i}\left(x-x_{i-0.5}\right) / h\right),
\end{aligned}
$$

where $a_{i}$ and $b_{i}$ are as defined in (5.2). Using $\left(1, x-x_{i-0.5}\right)_{i}=0$, we get

$$
\begin{aligned}
(k-\bar{k} & \left., q \exp (-\bar{D} / \varepsilon) G_{j}\right) \\
& =\sum_{i=1}^{N} s\left(x_{i}\right)\left(a_{i}-b_{i}\right) \exp \left(-\bar{D}\left(x_{i-0.5}\right) / \varepsilon\right) \sinh \left(\alpha_{i}\right) \tau_{i}^{*}+O\left(h^{2}\right),
\end{aligned}
$$

where $\tau_{i}^{*}=\left(x-x_{i-0.5},\left\{\sinh \left(2 \alpha_{i}\left(x-x_{i-0.5}\right) / h\right)\right\} / 2 \sinh \left(\alpha_{i}\right)\right)_{i}\left[\right.$ use $e^{-y}=\cosh (y)$ $-\sinh (y)$ and (5.4)]. Let

$$
c_{i}=\left(a_{i}-b_{i}\right) \exp \left(-\bar{D}\left(x_{i-0.5}\right) / \varepsilon\right) \sinh \left(\alpha_{i}\right) .
$$

With this notation,

$$
\left(k-\bar{k}, q \exp (-\bar{D} / \varepsilon) G_{j}\right)=\sum_{i=1}^{N} s\left(x_{i}\right) c_{i} \tau_{i}^{*}+O\left(h^{2}\right) .
$$

From the definition of $a_{i}$ and $b_{i}$,

$$
c_{i}=\exp \left(-\bar{D}\left(x_{i}\right) / \varepsilon\right) G_{j}\left(x_{i}\right)-\exp \left(-\bar{D}\left(x_{i-1}\right) / \varepsilon\right) G_{j}\left(x_{i-1}\right) \equiv \eta_{i}-\eta_{i-1} .
$$

Consequently,

$$
\sum_{i=1}^{N} s\left(x_{i}\right) c_{i} \tau_{i}^{*}=\sum_{i=1}^{N} s\left(x_{i}\right)\left(\eta_{i}-\eta_{i-1}\right) \tau_{i}^{*}=\sum_{i=1}^{N-1}\left(s\left(x_{i}\right) \tau_{i}^{*}-s\left(x_{i+1}\right) \tau_{i+1}^{*}\right) \eta_{i}
$$


since $G_{j}\left(x_{0}\right)=G_{j}\left(x_{N}\right)=0$. Note that Lemma 5.2 can be applied to $\tau_{i}^{*}$ and that $\left|\eta_{i}\right| \leqslant\left|G_{j}\left(x_{i}\right)\right|$ holds. Hence we get as in part (i),

$$
\left|\sum_{i=1}^{N} s\left(x_{i}\right) c_{i} \tau_{i}^{*}\right| \leqslant C h^{2}
$$

which completes the proof of (ii).

(iii) We have

$$
\left(k-\bar{k}, q \exp (-(D(1)-D(x)) / \varepsilon) G_{j}(x)\right)=\left(k_{1}-\bar{k}_{1}, q_{1} \exp \left(-D_{1}(t) / \varepsilon\right) F_{j}(t)\right),
$$

where $k_{1}(t)=k(1-t), \bar{k}_{1}(t)=\bar{k}(1-t), q_{1}, r_{1}$, and $p_{1}$ defined similarly,

$$
D_{1}(t)=\int_{0}^{t}\left(r_{1}(s) / p_{1}(s)\right)^{1 / 2} d s
$$

and $F_{j}(t)$ is defined as in the last part of Theorem 4.3. Since $F_{j}(t)$ satisfies the same sort of differential equation as $G_{j}(x)$ did, we can use (ii) to get the result.

(iv) From (5.2), on the interval $\left(x_{i-1}, x_{i}\right)$,

$$
G_{j}^{\prime}(x)=\left(\alpha_{i} a_{i} / h\right) \cosh \left(\alpha_{i}\left(x-x_{i-0.5}\right) / h\right)+\left(\alpha_{i} b_{i} / h\right) \sinh \left(\alpha_{i}\left(x-x_{i-0.5}\right) / h\right) \text {. }
$$

Thus

$$
\begin{aligned}
& \left|\left(\varepsilon^{2}(k-\bar{k}), q G_{j}^{\prime}\right)\right| \\
& \quad=\left|\sum_{i=1}^{N} \varepsilon^{2} h^{-1} \alpha_{i} s\left(x_{i}\right) \tau_{i}\left\{G_{j}\left(x_{i}\right)+G_{j}\left(x_{i-1}\right)\right\} \tanh \left(\alpha_{i} / 2\right)\right|+O\left(h^{2}\right) \\
& \quad \leqslant C h^{2} \varepsilon \sum_{i=1}^{N}\left|G_{j}\left(x_{i}\right)+G_{j}\left(x_{i-1}\right)\right|\left(1-e^{-\alpha_{i}}\right)+O\left(h^{2}\right),
\end{aligned}
$$

since, by Lemma 5.2, $\left|\tau_{i}\right| \leqslant C h^{2}$. From Theorem 4.2, we have

$$
\left|G_{j}\left(x_{i-1}\right)+G_{j}\left(x_{i}\right)\right| \leqslant C \varepsilon^{-1} \begin{cases}\exp \left(-\gamma\left|x_{j}-x_{i}\right| / \varepsilon\right), & i \leqslant j \\ \exp \left(-\gamma\left|x_{i-1}-x_{j}\right| / \varepsilon\right), & i>j .\end{cases}
$$

Using this, we get the result.

(v) Follow the argument in (ii), replacing $G_{j}$ by $\varepsilon G_{j}^{\prime}$, and note that

$$
\varepsilon G_{j}^{\prime}(x)=\left(\varepsilon \alpha_{i} /(2 h)\right)\left\{\left(a_{i}+b_{i}\right) e^{\alpha\left(x-x_{i-0.5}\right) / h}+\left(a_{i}-b_{i}\right) e^{-\alpha_{i}\left(x-x_{i-0.5}\right) / h}\right\} .
$$

(vi) Use the transformation given in (iii) and then use (v).

(vii) Write $w(x)=w\left(x_{i-0.5}\right)+\left(w(x)-w\left(x_{i-0.5}\right)\right)$. Thus,

$$
\begin{aligned}
\left(\varepsilon^{2}(k-\bar{k}), w G_{j}^{\prime}\right)= & \sum_{i=1}^{N} k^{\prime}\left(x_{i-0.5}\right)\left(w(x)-w\left(x_{i-0.5}\right),\left(x-x_{i-0.5}\right) \varepsilon^{2} G_{j}^{\prime}\right)_{i} \\
& +O\left(h^{2}\right) \text { by (iv). }
\end{aligned}
$$

Therefore,

$$
\left|\left(k-\bar{k}, \varepsilon^{2} w G_{j}^{\prime}\right)\right| \leqslant C h^{2} \max \left|w^{\prime}(x)\right|\left(1, \varepsilon^{2}\left|G_{j}^{\prime}\right|\right)+C h^{2} .
$$

Now $\left(1, \varepsilon\left|G_{j}^{\prime}\right|\right) \leqslant C$ by Theorem 4.3 . Thus

$$
\left|\left(k-\bar{k}, \varepsilon^{2} w G_{j}^{\prime}\right)\right| \leqslant C h^{2} \varepsilon \max \left|w^{\prime}(x)\right|+C h^{2} \leqslant C h^{2} .
$$


THEOREM 5.4. Let $y(x)$ be the solution of problem (1.1). Let $u(x)$ be the approximation defined in (3.2) and (3.3). Then

$$
\max _{0 \leqslant i \leqslant N}\left|y\left(x_{i}\right)-u\left(x_{i}\right)\right| \leqslant C h^{2} .
$$

Proof. Substitute the asymptotic expansion given in Lemma 2.7 into the closed form expression for the nodal error given in Theorem 5.1 and use Lemma 5.3 to bound each term. The nine terms involved are

$$
\begin{aligned}
y\left(x_{j}\right)-u\left(x_{j}\right)= & \left(f-\bar{f}, G_{j}\right)+\left(r-\bar{r},-(f / r) G_{j}\right) \\
& +\varepsilon^{2}\left(p-\bar{p},-(f / r)^{\prime} G_{j}^{\prime}\right)+\left(r-\bar{r}, K_{1} e^{-D(x) / \varepsilon} G_{j}\right) \\
& -\varepsilon\left(p-\bar{p}, K_{1}(r / p)^{1 / 2} e^{-D(x) / \varepsilon} G_{j}^{\prime}\right)+\left(r-\bar{r}, K_{2} e^{-(D(1)-D(x)) / \varepsilon} G_{j}\right) \\
& +\varepsilon\left(p-\bar{p}, K_{2}(r / p)^{1 / 2} e^{-(D(1)-D(x)) / \varepsilon} G_{j}^{\prime}\right)+\left(r-\bar{r}, \varepsilon R_{0} G_{j}\right) \\
& +\varepsilon^{2}\left(p-\bar{p}, R_{0}^{\prime} G_{j}^{\prime}\right) .
\end{aligned}
$$

The last two integrals need the bounds given in Lemma 2.8 .

6. A Uniform Global Error Estimate. As in O'Riordan [9], we choose the trial functions in such a way that, "close" to a boundary layer, they mirror the shape and direction of that boundary layer. For notational convenience, we will take $N$ to be even. Define the trial functions $\left\{\phi_{i}\right\}_{i=0}^{N}$ by

$$
\begin{array}{ll}
\bar{L}_{1} \phi_{i} \equiv \varepsilon^{2} \bar{p} \phi_{i}^{\prime \prime}+\varepsilon(\overline{r p})^{1 / 2} \phi_{i}^{\prime}=0 & \text { on }[0,0.5] \cap[0,1]^{*}, \\
\bar{L}_{2} \phi_{i} \equiv \varepsilon^{2} \bar{p} \phi_{i}^{\prime \prime}-\varepsilon(\overline{r p})^{1 / 2} \phi_{i}^{\prime}=0 & \text { on }[0.5,1] \cap[0,1]^{*}, \\
\phi_{i}\left(x_{j}\right)=\delta_{i, j}, \quad j=0, \ldots, N &
\end{array}
$$

THEOREM 6.1. If the trial and test functions are chosen as in (6.1) and (3.1), respectively, then

$$
\|y-u\|_{\infty} \leqslant C h
$$

where $y$ is the solution of (1.1) and $u$ is the approximation defined in (3.2) and (3.3).

Proof. (a) Let $x \in\left(x_{i-1}, x_{i}\right)$, where $i \leqslant N / 2$ is fixed.

$$
\begin{aligned}
\bar{L}_{1} y & =\varepsilon^{2} \bar{p} y^{\prime \prime}+\varepsilon(\overline{r p})^{1 / 2} y^{\prime} \\
& =\varepsilon^{2} p y^{\prime \prime}+\varepsilon(r p)^{1 / 2} y^{\prime}+\varepsilon^{2}(\bar{p}-p) y^{\prime \prime}-(\sqrt{\overline{r p}}-\sqrt{r p}) \varepsilon y^{\prime} \\
& =f+r y+\varepsilon(r p)^{1 / 2} y^{\prime}-\varepsilon^{2} p^{\prime} y^{\prime}+\varepsilon^{2}(\bar{p}-p) y^{\prime \prime}+(\sqrt{\overline{r p}}-\sqrt{r p}) \varepsilon y^{\prime} .
\end{aligned}
$$

Using the bounds from Corollary 2.6,

$$
\begin{aligned}
&\left|\bar{L}_{1} y\right| \leqslant\left|f+r y+\varepsilon(r p)^{1 / 2} y^{\prime}\right|+C \varepsilon+C h e^{-\delta_{1} x / \varepsilon} \\
& \text { since } \exp \left(-\delta_{1}(1-x) / \varepsilon\right) \leqslant \exp \left(-\delta_{1} x / \varepsilon\right) \text { for } x \leqslant 0.5 .
\end{aligned}
$$

Substituting the asymptotic expansion for $y$ from Lemma 2.7, we get

$$
f+r y+\varepsilon(r / p)^{1 / 2} y^{\prime}=2 K_{2} r e^{-(D(1)-D(x)) / \varepsilon}+\varepsilon k(x),
$$

where $|k(x)|=\left|\left\{\varepsilon R_{0}^{\prime}-(f / r)^{\prime}\right\}(r p)^{1 / 2}+r R_{0}\right| \leqslant C$. Therefore,

$$
\begin{aligned}
\left|f+r y+\varepsilon(r p)^{1 / 2} y^{\prime}\right| & \leqslant C \varepsilon\left(1+\varepsilon^{-1} e^{-\gamma / 2 \varepsilon}\right) \quad \text { since } x \leqslant 0.5 \\
& \leqslant C \varepsilon .
\end{aligned}
$$


Thus $\left|\bar{L}_{1} y\right| \leqslant C \varepsilon+C h e^{-\delta_{1} x / \varepsilon}$. Set $z(x)=C\left(x-x_{i}-h\right)-C h e^{-\gamma x / \varepsilon} \pm(y-u)$, where the positive constants $C, \gamma$ are to be chosen. Now for $C$ sufficiently large, Theorem 5.4 implies that $z\left(x_{i-1}\right) \leqslant 0$ and $z\left(x_{i}\right) \leqslant 0$. Also,

$$
\bar{L}_{1} z=C \varepsilon(\overline{r p})^{1 / 2}+\bar{p} \operatorname{Ch} \gamma\left\{(\bar{r} / \bar{p})^{1 / 2}-\gamma\right\} e^{-\gamma x / \varepsilon} \pm \bar{L}_{1} y .
$$

Now $\left|\bar{L}_{1} y\right| \leqslant C \varepsilon+C h e^{-\delta_{1} x / \varepsilon}$. Hence we can choose $C$ and $\gamma$ such that $\bar{L}_{1} z>0$. Since $\bar{L}_{1}$ satisfies a maximum principle (see Protter and Weinberger [8]), we have $z \leqslant 0$ on $\left[x_{i-1}, x_{i}\right]$, i.e., $|y-u| \leqslant C h$ on $\left[x_{i-1}, x_{i}\right], i \leqslant N / 2$.

(b) Similarly for $x \in\left(x_{i-1}, x_{i}\right)$, where $i>N / 2$ is fixed, use the maximum principle for $\bar{L}_{2}$ applied to the barrier function

$$
z(x)=C\left(x_{i}-x-2 h\right)-C h e^{-\gamma(1-x) / \varepsilon} \pm(y-u) \text { for } x \in\left[x_{i-1}, x_{i}\right] .
$$

7. Numerical Experiments. In this section, we present some numerical results which illustrate Theorem 5.4. All computations were performed in Fortran double precision on a VAX 11/780 at University College, Cork. The authors are indebted to Dr. Jeanne Ng-Stynes for these results.

The difference scheme (3.3) may be written in the form

$$
\varepsilon^{2} h^{-2}\left\{a_{j}^{-} u_{j-1}+a_{j}^{0} u_{j}+a_{j}^{+} u_{j+1}\right\}=c_{j}^{-} f_{j-1}+c_{j}^{0} f_{j}+c_{j}^{+} f_{j+1},
$$

where

$$
\begin{aligned}
& a_{j}^{-}=\bar{p}_{j} \sigma\left(\alpha_{j}\right), \quad a_{j}^{+}=\bar{p}_{j+1} \sigma\left(\alpha_{j+1}\right), \quad a_{j}^{0}=-\bar{p}_{j} \eta\left(\alpha_{j}\right)-\bar{p}_{j+1} \eta\left(\alpha_{j+1}\right), \\
& c_{j}^{-}=\sigma\left(\alpha_{j}\right)\left(2 \sigma\left(\alpha_{j} / 2\right)\right)^{-2}, \quad c_{j}^{+}=\sigma\left(\alpha_{j+1}\right)\left(2 \sigma\left(\alpha_{j+1} / 2\right)\right)^{-2}, \\
& c_{j}^{0}=c_{j}^{-}+c_{j}^{+}, \quad \alpha_{j}=\left(\bar{r}_{j} / \bar{p}_{j}\right)^{1 / 2} h \varepsilon^{-1}, \\
& \bar{p}_{j}=\left(p\left(x_{j-1}\right)+p\left(x_{j}\right)\right) / 2, \quad \bar{r}_{j}=\left(r\left(x_{j-1}\right)+r\left(x_{j}\right)\right) / 2,
\end{aligned}
$$

and

$$
\sigma(x)=x / \sinh (x), \quad \eta(x)=x \operatorname{coth}(x) .
$$

Example 7.1. We applied this difference scheme to problem (1.1), with

$$
\begin{gathered}
p(x)=1+x^{2}, \quad r(x)=\cos (x)(3-x)^{-3}, \\
f(x)=4\left(3 x^{2}-3 x+1\right)\left((x-0.5)^{2}+2\right), \quad y(0)=-1, \quad y(1)=0
\end{gathered}
$$

(see [2, p. 231]). The results are given in Table 1. The errors (EMAX) and the rates of convergence (RATE) are based on the double mesh principle (see [2, p. 223]). We define, for $N=8,16, \ldots, 512$,

$$
\operatorname{EMAX}=\max _{0 \leqslant j \leqslant N}\left|u_{j}^{N}-u_{2 j}^{2 N}\right| \text { and } \operatorname{RATE}=\left\{\ln \left(E^{1}\right)-\ln \left(E^{2}\right)\right\} / \ln (2),
$$

where $E^{1}$ and $E^{2}$ correspond to EMAX for $h=1 / N$ and $h=1 / 2 N$, respectively.

Example 7.2. We also applied this difference scheme to problem (1.1), with

$$
\begin{gathered}
p(x)=1, \quad r(x)=\{4+4 \varepsilon(1+x)\} /(1+x)^{4}, \\
f(x)=\left\{-4 /(1+x)^{4}\right\}\left[\left(1+\varepsilon(1+x)+4 \pi^{2} \varepsilon^{2}\right) \cos (2 \pi t)-2 \pi \varepsilon^{2}(1+x) \sin (2 \pi t)\right. \\
\left.+3(1+\varepsilon(1+x)) e^{-1 / \varepsilon}\left(1-e^{-1 / \varepsilon}\right)^{-1}\right], \\
y(0)=2.0, \quad y(1)=-1.0
\end{gathered}
$$


TABLE 1

RATE(EMAX)

\begin{tabular}{|c|l|l|l|l|l|l|}
\hline$N \quad \varepsilon$ & $1.0 \mathrm{E}-0.5$ & $1.0 \mathrm{E}-1.0$ & $1.0 \mathrm{E}-1.5$ & $1.0 \mathrm{E}-2.0$ & $1.0 \mathrm{E}-3.0$ & $1.0 \mathrm{E}-4.0$ \\
\hline 8 & 1.98 & 1.99 & 2.02 & 2.20 & 1.74 & 1.73 \\
& $(9.6 \mathrm{E}-2)$ & $(7.1 \mathrm{E}-1)$ & $(1.9 \mathrm{E}+0)$ & $(3.4 \mathrm{E}+0)$ & $(5.5 \mathrm{E}+0)$ & $(5.5 \mathrm{E}+0)$ \\
16 & 2.00 & 2.00 & 2.01 & 2.07 & 1.99 & 1.86 \\
& $(2.4 \mathrm{E}-2)$ & $(1.8 \mathrm{E}-1)$ & $(4.7 \mathrm{E}-1)$ & $(7.5 \mathrm{E}-1)$ & $(1.7 \mathrm{E}+0)$ & $(1.7 \mathrm{E}+0)$ \\
32 & 2.00 & 2.00 & 2.00 & 2.02 & 2.34 & 1.93 \\
& $(6.1 \mathrm{E}-3)$ & $(4.5 \mathrm{E}-2)$ & $(1.2 \mathrm{E}-1)$ & $(1.8 \mathrm{E}-1)$ & $(4.2 \mathrm{E}-1)$ & $(4.6 \mathrm{E}-1)$ \\
64 & 2.00 & 2.00 & 2.00 & 2.00 & 2.24 & 1.97 \\
& $(1.5 \mathrm{E}-3)$ & $(1.1 \mathrm{E}-2)$ & $(2.9 \mathrm{E}-2)$ & $(4.4 \mathrm{E}-2)$ & $(8.2 \mathrm{E}-2)$ & $(1.2 \mathrm{E}-1)$ \\
128 & 2.00 & 2.00 & 2.00 & 2.00 & 2.10 & 2.06 \\
& $(3.8 \mathrm{E}-4)$ & $(2.8 \mathrm{E}-3)$ & $(7.3 \mathrm{E}-3)$ & $(1.1 \mathrm{E}-2)$ & $(1.7 \mathrm{E}-2)$ & $(3.1 \mathrm{E}-2)$ \\
256 & 2.00 & 2.00 & 2.00 & 2.00 & 2.03 & 2.31 \\
& $(9.5 \mathrm{E}-5)$ & $(7.0 \mathrm{E}-4)$ & $(1.8 \mathrm{E}-3)$ & $(2.7 \mathrm{E}-3)$ & $(4.1 \mathrm{E}-3)$ & $(7.4 \mathrm{E}-3)$ \\
512 & 2.00 & 2.00 & 2.00 & 2.00 & 2.01 & 2.32 \\
& $(2.4 \mathrm{E}-5)$ & $(1.7 \mathrm{E}-4)$ & $(4.5 \mathrm{E}-4)$ & $(6.9 \mathrm{E}-4)$ & $(9.9 \mathrm{E}-4)$ & $(1.5 \mathrm{E}-3)$ \\
\hline
\end{tabular}

TABLE 2

$\operatorname{RATE}(\mathrm{EMAX})$

\begin{tabular}{|c|l|l|l|l|l|l|}
\hline$N$ & 1 & $h^{* *} 0.25$ & $h^{* *} 0.5$ & $h^{* *} 0.75$ & $h^{* *} 1$ & $h^{* *} 1.5$ \\
\hline 8 & 1.96 & 2.02 & 2.08 & 2.08 & 1.95 & 1.79 \\
& $(4.2 \mathrm{E}-1)$ & $(3.8 \mathrm{E}-1)$ & $(3.3 \mathrm{E}-1)$ & $(2.8 \mathrm{E}-1)$ & $(2.5 \mathrm{E}-1)$ & $(2.6 \mathrm{E}-1)$ \\
16 & 1.97 & 2.02 & 2.12 & 2.05 & 1.95 & 1.76 \\
& $(1.1 \mathrm{E}-1)$ & $(9.5 \mathrm{E}-2)$ & $(7.8 \mathrm{E}-2)$ & $(6.6 \mathrm{E}-2)$ & $(6.4 \mathrm{E}-2)$ & $(7.7 \mathrm{E}-2)$ \\
32 & 2.00 & 2.07 & 2.10 & 1.98 & 1.97 & 1.56 \\
& $(2.7 \mathrm{E}-2)$ & $(2.3 \mathrm{E}-2)$ & $(1.8 \mathrm{E}-2)$ & $(1.6 \mathrm{E}-2)$ & $(1.7 \mathrm{E}-2)$ & $(2.3 \mathrm{E}-2)$ \\
64 & 2.00 & 2.07 & 2.06 & 1.98 & 1.69 & 1.82 \\
& $(6.9 \mathrm{E}-3)$ & $(5.6 \mathrm{E}-3)$ & $(4.2 \mathrm{E}-3)$ & $(4.0 \mathrm{E}-3)$ & $(4.2 \mathrm{E}-3)$ & $(7.6 \mathrm{E}-3)$ \\
128 & 2.00 & 2.08 & 2.01 & 1.99 & 1.80 & 1.92 \\
& $(1.7 \mathrm{E}-3)$ & $(1.3 \mathrm{E}-3)$ & $(1.0 \mathrm{E}-3)$ & $(1.0 \mathrm{E}-3)$ & $(1.3 \mathrm{E}-3)$ & $(2.2 \mathrm{E}-3)$ \\
256 & 2.00 & 2.07 & 1.99 & 1.82 & 1.91 & 1.96 \\
& $(4.3 \mathrm{E}-4)$ & $(3.1 \mathrm{E}-4)$ & $(2.5 \mathrm{E}-4)$ & $(2.6 \mathrm{E}-4)$ & $(3.7 \mathrm{E}-4)$ & $(5.7 \mathrm{E}-4)$ \\
512 & 2.00 & 2.07 & 1.99 & 1.83 & 1.94 & 1.98 \\
& $(1.1 \mathrm{E}-4)$ & $(7.4 \mathrm{E}-5)$ & $(6.3 \mathrm{E}-5)$ & $(7.3 \mathrm{E}-5)$ & $(1.0 \mathrm{E}-4)$ & $(1.5 \mathrm{E}-4)$ \\
\hline
\end{tabular}

(see [10, p. 81]). The results are given in Table 2 . In this example, the analytic solution can be computed. Thus we define

$$
\text { EMAX }=\max _{0 \leqslant j \leqslant N}\left|y\left(x_{j}\right)-u\left(x_{j}\right)\right| \text { and } \mathrm{RATE}=\left\{\ln \left(E^{1}\right)-\ln \left(E^{2}\right)\right\} / \ln (2),
$$

where $E^{1}$ and $E^{2}$ correspond to EMAX for $h=1 / N$ and $h=1 / 2 N$, respectively.

Department of Science

Regional Technical College

Dundalk, Ireland

Department of Mathematics

University College

Cork, Ireland

1. I. P. Boglaev, "A variational difference scheme for a boundary value problem with a small parameter in the highest derivative," U.S.S.R. Comput. Math. and Math. Phys., v. 21, 1981, no. 4, pp. $71-81$.

2. E. P. Doolan, J. J. H. Miller \& W. H. A. Schilders, Uniform Numerical Methods for Problems with Initial and Boundary Layers, Boole Press, Dublin, 1980. 
3. P. P. N. DE Groen, "A finite element method with a large mesh width for a stiff two-point boundary value problem," J. Comput. Appl. Math., v. 7, 1981, pp. 3-15.

4. A. F. Hegarty, J. J. H. Miller \& E. O’Riordan, "Uniform second order difference schemes for singular perturbation problems," Boundary and Interior Layers-Computational and Asymptotic Methods (J. J. H. Miller, ed.), Boole Press, Dublin, 1980, pp. 301-305.

5. P. W. Hemker, A Numerical Study of Stiff Two-point Boundary Value Problems, Mathematical Centre Tracts, No. 80, Amsterdam, 1977.

6. J. J. H. Miller, "On the convergence, uniformly in $\varepsilon$, of difference schemes for a two-point boundary value singular perturbation problem," Numerical Analysis of Singular Perturbation Problems (P. W. Hemker \& J. J. H. Miller, eds.), Academic Press, New York, 1979, pp. 467-474.

7. K. Ninima, "On a three-point difference scheme for a singular perturbation problem without a first derivative term I," Mem. Numer. Math., v. 7, 1980, pp. 1-10.

8. M. A. Protter \& H. F. Weinberger, Maximum Principles in Differential Equations, Prentice-Hall, Englewood Cliffs, N. J., 1967.

9. E. O'Riordan, Finite Element Methods for Singularly Perturbed Problems, Ph.D. thesis, School of Mathematics, Trinity College, Dublin, 1982.

10. A. H. Schatz \& L. B. Wahlbin, "On the finite element method for singularly perturbed reaction-diffusion problems in two and one dimensions," Math. Comp., v. 40, 1983, pp. 47-89.

11. G. I. SHISHKIN, "A difference scheme on a non-uniform mesh for a differential equation with a small parameter in the highest derivative," U.S.S. R. Comput. Math. and Math. Phys., v. 23, no. 3, 1983, pp. 59-66.

12. M. STYNES \& E. O'RIORDAN, “A superconvergence result for a singularly perturbed boundary value problem," BAIL III-Proc. Third International Conference on Boundary and Interior Layers (J. J. H. Miller, ed.), Boole Press, Dublin, 1984, pp. 309-313.

13. R. S. VARgA, Matrix Iterative Analysis, Prentice-Hall, Englewood Cliffs, N. J., 1962. 